Detween the medial geniculate body and the lateral nucleus of the amygdala. Exp. Brain Res. 105, 87-100 (1995)

16. Maren, S., Aharonov, G., Stote, D. L. \& Fanselow, M. S. N-methyl-D-aspartate receptors in the basolateral amygdala are required for both acquisition and expression of conditioned fear in rats. Behav. Neurosci. 110, 1365-1374 (1996).

17. Pavlov, I. P. Conditioned Reflexes: An Investigation of the Physiological Activity of the Cerebral Cortex (Dover, New York, 1927).

18. Rescorla, R. A. Pavlovian Second-order Conditioning: Studies in Associative Learning (Erlbaum, Hillsdale, NJ, 1980)

19. Kim, M., Campeau, S., Falls, W. A. \& Davis, M. Infusion of the non-NMDA receptor antagonist CNQX into the amygdala blocks expression of fear-potentiated startle. Behav. Neural Biol. 59, 5-8 (1993).

20. Campeau, S. \& Davis, M. Involvement of the central nucleus and basolateral complex of the amygdal in fear conditioning measured with fear-potentiated startle in rats trained concurrently with auditory and visual conditioned stimuli. J. Neurosci. 15, 2301-2311 (1995).

21. Romanski, L. M., Clugnet, M.-C., Bordi, F. \& LeDoux, J. E. Somatosensory and auditory convergence in the lateral nucleus of the amygdala. Behav. Neurosci. 107, 444-450 (1993).

22. Campeau, S. et al. Induction of the c-fos proto-oncogene in the rat amygdala during unconditioned and conditioned fear. Brain Res. 565, 349-352 (1991).

23. Falls, W. F., Miserendino, M. J. D. \& Davis, M. Extinction of fear-potentiated startle: Blockade by infusion of an NMDA antagonist into the amygdala. J. Neurosci. 12, 854-863 (1992)

24. Campeau, S. \& Davis, M. Involvement of subcortical and cortical afferents to the lateral nucleus of the amygdala in fear conditioning measured with fear-potentiated startle in rats trained concurrently with auditory and visual conditioned stimuli. J. Neurosci. 15, 2312-2327 (1995).

25. Romanski, L. M. \& LeDoux, J. E. Equipotentiality of thalamo-amygdala and thalamo-corticoamygdala circuits in auditory fear conditioning. J. Neurosci. 12, 4501-4509 (1992).

26. Li, X. F., Stutzmann, G. E. \& LeDoux, J. E. Convergent but temporally separated inputs to latera amygdala neurons from the auditory thalamus and auditory cortex use different postsynaptic receptors: In vivo intracellular and extracellular recordings in fear conditioning pathways. Learn. Mem. 3, 229-242 (1996)

27. Hatfield, T., Han, J.-S., Conley, M., Gallagher, M. \& Holland, P. C. Neurotoxic lesions of basolateral, but not central, amygdala interfere with Pavlovian second-order conditioning and reinforcer devaluation effects. J. Neurosci. 16, 5256-5265 (1996).

28. Everitt, B. J. \& Robbins, T. W. in The Amygdala: Neurobiological Aspects of Emotion, Memory, and Mental Dysfunction (ed. Aggleton, J. P.) 401-429 (Wiley-Liss, New York, 1992).

29. Willick, M. L. \& Kokkinides, L. Cocaine enhances the expression of fear-potentiated startle Evaluation of state-dependent extinction and the shock-sensitization of acoustic startle. Behav Neurosci. 109, 929-938 (1995).

30. Cassella, J. V. \& Davis, M. The design and calibration of a startle measurement system. Physiol. Behav 36, 377-383 (1986)

Acknowledgements. We thank Bill Falls, whose pilot studies suggested a role for NMDA receptors in acquisition of second-order conditioning and stimulated this investigation. This research was supported by grants from AFPSR and NIMH (to M.D.) and by an NIH individual NRSA from NIMH (to J.C.G.)

Correspondence and requests for materials should be addressed to J.C.G. (e-mail: jonathan.gewirtz@yale. edu).

\section{Transport, docking and exocytosis of single secretory granules in live chromaffin cells}

\section{J. A. Steyer, H. Horstmann \& W. Almers}

Max-Planck-Institut für medizinische Forschung, 69120 Heidelberg, Jahnstrasse 29, Germany

Neurons maintain a limited pool of synaptic vesicles which are docked at active zones and are awaiting exocytosis ${ }^{1-4}$. By contrast, endocrine cells releasing large, dense-core secretory granules have no active zones, and there is disagreement about the size ${ }^{5}$ and even the existence ${ }^{6}$ of the docked pool. It is not known how, and how rapidly, secretory vesicles are replaced at exocytic sites in either neurons or endocrine cells. By using electron microscopy, we have now been able to identify a pool of docked granules in chromaffin cells that is selectively depleted when cells secrete. With evanescent-wave fluorescence microscopy ${ }^{7}$, we observed single granules undergoing exocytosis and leaving behind patches of bare plasmalemma. Fresh granules travelled to the plasmalemma at a top speed of $114 \mathrm{~nm} \mathrm{~s}^{-1}$, taking an average of $6 \mathrm{~min}$ to arrive. On arrival, their motility diminished 4-fold, probably as a result of docking. Some granules detached and returned to the cytosol. We conclude that a large pool of docked granules turns over slowly, that granules move actively to their docking sites, that docking is reversible, and that the 'rapidly releasable pool' measured electrophysiologically represents a small subset of docked granules.
Even in chromaffin cells, a population of docked dense-core granules can be morphologically identified if chemical fixation is avoided (Fig. 1a). We measured the closest distance between granules and the plasmalemma and plotted the results as a histogram (Fig. 1b). Granules populated the cytosol at a constant density, although those touching the plasmalemma were more numerous. In terms of granules per $\mu \mathrm{m}^{2}$ (Fig. 1 legend), the cytosolic density of granules (dashed line) is only $5 \%$ of the density of those touching the plasmalemma. The remaining 95\% must be bound to the plasmalemma, or docked. Their number in a resting cell was $1.68 \pm 0.11 \mu^{-2}$ (5 experiments) or $1,010 \pm 80$ in a typical cell with a $600 \mu \mathrm{m}^{2}$ surface area. When cells were stimulated by elevating the external potassium ion concentration $\left(\left[\mathrm{K}^{+}\right]\right)$, granules were lost by exocytosis (Fig. 1c). Granules touching the plasmalemma were lost selectively (Fig. 1d); $53 \pm 11 \%$ of them were lost during 2 minutes of stimulation (5 experiments). Selective depletion of docked granules over $2 \mathrm{~min}$ is not expected if cytosolic and docked granules reach equilibrium over that time. For instance, the 'rapidly releasable pool' of secretory vesicles in chromaffin cells ${ }^{8}$ and neurons $s^{9,10}$ is replenished with time constants of only $10 \mathrm{~s}$. In chromaffin cells, this evidently can happen without docking new granules.

The dynamics of these morphological changes was studied in living cells ${ }^{24}$ by fluorescence microscopy. Cells were allowed to attach to glass coverslips, then stained briefly with acridine orange and washed thoroughly. For viewing single granules beneath the plasmalemma, confocal microscopy had insufficient depth resolution and, most importantly, could image single granules only at illumination levels that caused rapid bleaching and photoxicity. To image a thin layer of cytosol with less intense illumination, we used a method not previously applied in neurobiology. Fluorescence was excited selectively in a 300-nm-thin layer of cytosol immediately adjacent to the coverslip, using the evanescent wave set up by a laser beam as it suffered total reflection at the glass/cell interface ${ }^{7}$. Typical images (Fig. 1e, f) show scattered fluorescent spots. Because granules are by far the most abundant organelle near the plasmalemma (Fig. 1a), most spots must represent granules close to the plasmalemma where it adheres to the coverslip.

Stimulation with high $\left[\mathrm{K}^{+}\right]$caused fluorescent spots to disappear (Fig. 1f) as exocytosing granules lost their dye. Granules were lost in patches, consistent with earlier reports of localized secretion ${ }^{11}$. The patches did not arise because the plasmalemma buckled and moved out of the evanescent wave, because in simultaneous interference reflection images (Fig. 1g, h) the 'footprint' of the cell on the coverglass remained dark; the footprint would have brightened had the plasmalemma detached. During a 2-min exposure to high $\left[\mathrm{K}^{+}\right]$, $34 \pm 6 \%$ of the granules were lost (15 cells). This is less than the $53 \%$ lost in Fig. 1b, c, presumably because the evanescent wave also excites fluorescence in some granules that do not touch the plasmalemma and hence cannot perform exocytosis. Comparison of the two values suggests that in a resting cell, $34 / 53=64 \%$ of the fluorescent spots are secretory granules touching the plasmalemma.

The cells we studied had $1.3 \pm 0.4$ fluorescent spots per $\mu \mathrm{m}^{2}(24$ cells). If only $64 \%$ represent granules touching the plasmalemma, we see fewer such granules $\left(0.8\right.$ per $\left.\mu \mathrm{m}^{2}\right)$ than in electron micrographs. Although plasmalemma adhering to glass may carry fewer granules, we believe that two other factors are also important: first, we tended to select cells with lower granule density (see Methods); second, granules in electron micrographs varied 2-3-fold in diameter and hence more than 8 -fold in volume. If granules carry dye and fluoresce in proportion to their volume, small ones will be overlooked if they are next to larger ones.

Four findings indicate that most of the fluorescent granules in Fig. 1e, f were lost through exocytosis. First, the effect was not seen when $\mathrm{Mg}^{2+}$ replaced external $\mathrm{Ca}^{2+}$ and $0.1 \mathrm{~mm} \mathrm{Cd}^{2+}$ was added ( $99 \pm 4 \%$ remaining after $2 \mathrm{~min}$ at high $\left[\mathrm{K}^{+}\right] ; 5$ cells). Second, fluorescent spots disappeared abruptly from one frame to the next 
(Fig. 2a, images 2, 3) even in recordings made at 1 frame per second (not shown). If instead, granules left the plasmalemma and moved into the cytosol, they would need a median time of $14 \mathrm{~s}$ to escape the evanescent wave and become invisible (see below). Third, a diffuse cloud of secreted dye often appeared briefly at the point where a granule disappeared (Fig. 2a, images 5, 6). Finally, recordings of single catecholamine quanta (Fig. 2c) revealed that significant secretion happened only during stimulation (Fig. 2b), as did the loss of granules (Fig. 2d). Secretion of quanta and loss of granules proceeded roughly proportionately to each other.
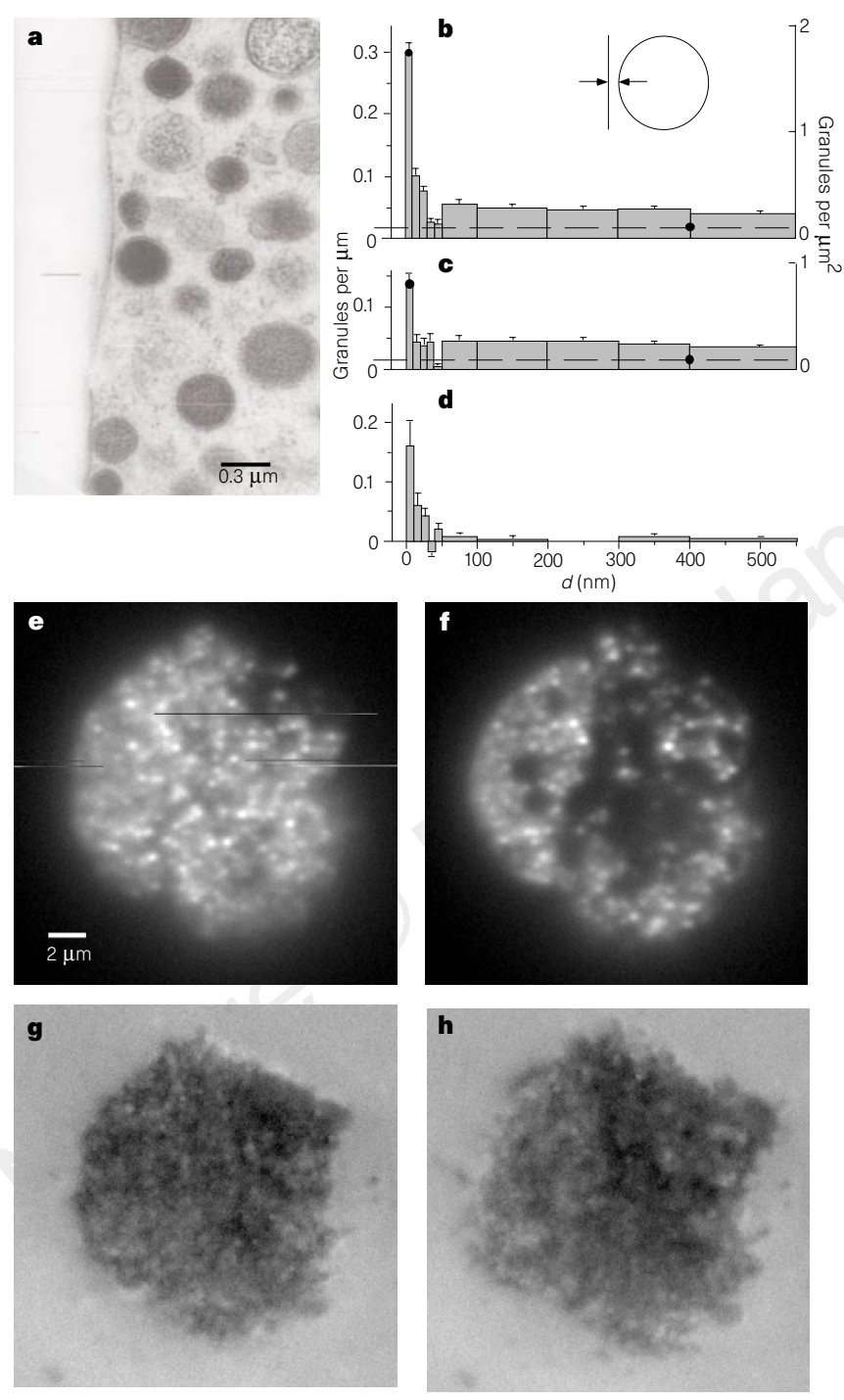

Figure 1 a, Unfixed, quickly frozen chromaffin cell showing plasmalemma (left) and the granules beneath. $\mathbf{b}$, Number of granules as a function of distance $d$ from the plasmalemma, measured as shown in the inset and calculated for $10-\mathrm{nm}$ bin width. Bars give granules per $\mu \mathrm{m}$ of plasmalemma; filled circles were converted into granules per $\mu \mathrm{m}^{2}$. The filled circle at $400 \mathrm{~nm}$ (dashed line) is the mean per 10$\mathrm{nm}$ bin for $150<d<600 \mathrm{~nm}$, and proportional to the cytosolic granule density $\left(0.11 \mu \mathrm{m}^{-2}\right.$ for a $10 \mathrm{~nm}$ layer, or $\left.10.5 \mu \mathrm{m}^{-3}\right)$. c. As in $\mathbf{b}$, but after 2 -min stimulation in a solution with high $\left[\mathrm{K}^{+}\right]$, expected to raise cytosolic $\left[\mathrm{Ca}^{2+}\right]$ by influx through plasmalemmal calcium channels. d, Granules lost during the 2-min stimulation (difference between $\mathbf{b}$ and $\mathbf{c}$ ). e-h, Light micrographs showing the region of a cell where it adheres to a glass coverslip. e, f, Evanescent-wave fluorescence micrographs: e, in normal buffer, and $\mathbf{f}$, after $2 \mathrm{~min}$ in high $\left[\mathrm{K}^{+}\right]$, as in $\mathbf{c .} \mathbf{g}, \mathbf{h}$ Interference reflection images taken within $1 \mathrm{~s}$ of $\mathbf{e}, \mathbf{f}$, respectively.
To determine how rapidly the depleted plasmalemma is repopulated with granules, we watched fluorescent spots appear after stimulation stopped (Fig. 3a, b). As dye had been absent from the bathing medium for about 1 hour, the new spots must represent stained granules that had moved from the cytosol into the evanescent wave and onto the plasmalemma (Fig. 3c). The plasmalemmal granule population was partially replenished with a time constant of $6 \mathrm{~min}$. This is $>10$-fold slower than at synapses, where endocytic vesicles become release-ready synaptic vesicles in only 30-60 s (hippocampal synapses ${ }^{12}$ ) or $15-30$ s (frog neuromuscular junction $\left.{ }^{13}\right)$.

The appearance of a single granule beneath the plasmalemma is shown in Fig. 4a; Fig. 4b plots the time course of fluorescence at the granule site. When the granule appeared, fluorescence rose above background and increased to a plateau as the granule reached the plasmalemma. As the fluorescence grew, the granule brightened continually, never reversing its course towards the plasmalemma over $14 \mathrm{~s}$. Such directed movement is not expected for a diffusion process and indicates that the granule moved actively. The granule ultimately docked successfully, because a second stimulus triggered its exocytosis (Fig. 4b, arrowhead); this happened $174 \mathrm{~s}$ after its arrival and $96 \mathrm{~s}$ after the second stimulus. Once at the plasmalemma, a granule evidently reaches fusion competence within minutes.

a
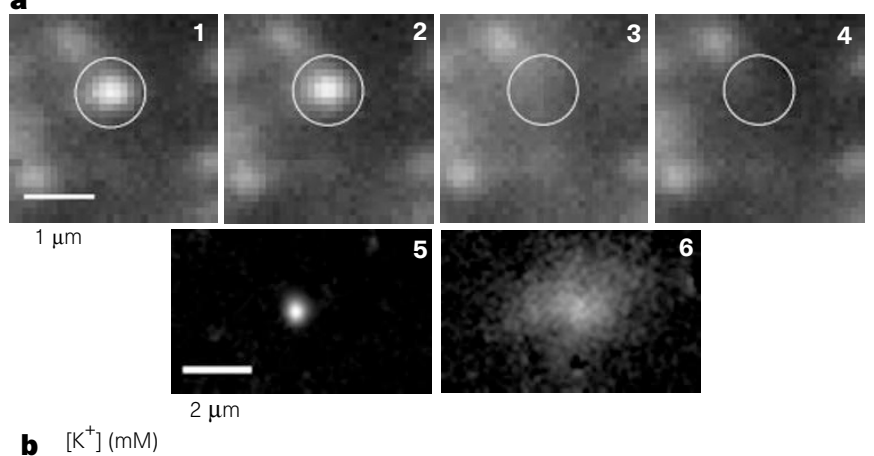

b $\quad\left[\mathrm{K}^{+}\right](\mathrm{mM})$

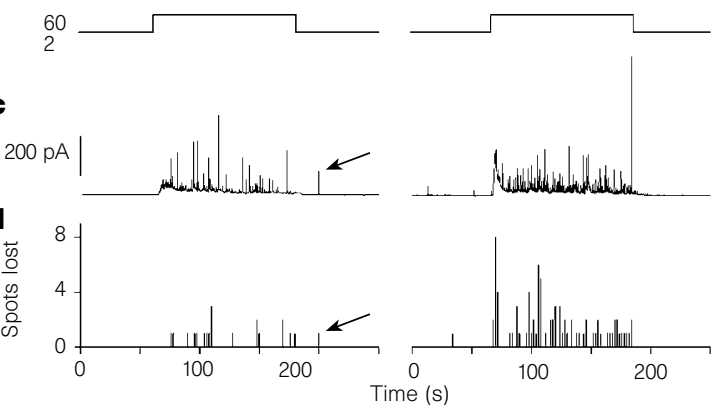

Figure 2 a, 1-4, Successive frames at $0.5 \mathrm{~s}^{-1}$ show a granule $(1,2)$ vanishing abruptly owing to exocytosis $(2,3)$; the granule and its last location are indicated by circles. Images 5, 6 are the same as 2, 3 after subtracting image 4. The summed glow of the cloud in image 6 was more than that from the granule before exocytosis. This is consistent with secretion against the coverslip, where the dye is most strongly excited by the evanescent wave. b-d, Behaviour of two other cells during a similar experiment. b. External $\left[\mathrm{K}^{+}\right]$was raised and lowered by a rapid perfusion system. c, Current spikes recorded by a carbon fibre near the cell; each represents a single quantum of released catecholamine ${ }^{23}$. Secretion starts when $\left[\mathrm{K}^{+}\right]$is raised and stops when it is lowered again. $\mathbf{d}$, Data from simultaneous video clips; number of granules lost in successive 2-s intervals plotted against time. One granule was located at the margin of the cell facing the carbon fibre, and its disappearance coincided with the release of a catecholamine quantum (arrow). Generally, however, carbon fibre and microscope sample different regions of the cell, hence the detected events lack one-to-one correspondence. 

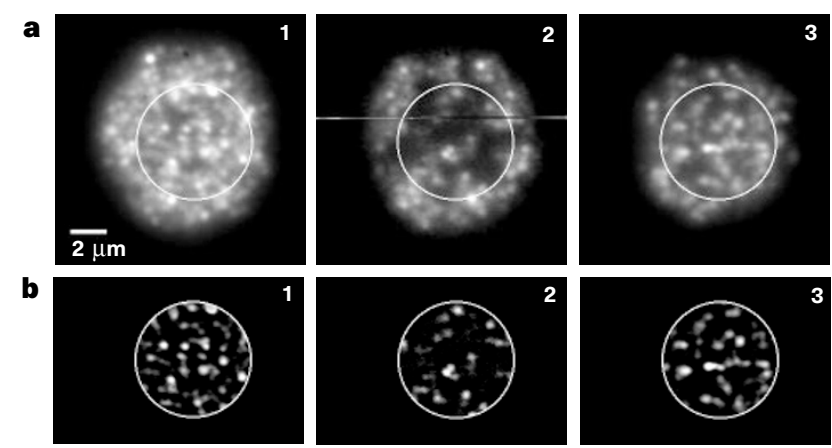

Figure $3 \mathbf{a}$, Images before (1), immediately after (2) and 20 min after the end of a 2min stimulation with elevated $\left[\mathrm{K}^{+}\right]$(3). b. Area marked by the circle in $\mathbf{a}$. To enhance contrast, we subtracted from each image a version that had been low-pass filtered at a spatial frequency of $1 / \mu \mathrm{m}$. c. Top, drawing showing coverslip with adherent portion of a cell; granules fluoresce (black) only within the evanescent wave (shaded area). Bottom, granule density within the circles in $\mathbf{b}$ normalized to the prestimulus value $\left(1.4 \pm 0.1 \mu \mathrm{m}^{-2}\right)$ and plotted as a function of time. High $\left[\mathrm{K}^{+}\right]$ applied within rectangle. Both the decline and recovery of granule density (to $69 \pm 9 \%$ in this dataset of 6 cells) varied strongly from cell to cell. To reduce effects of this variability on our estimate of the recovery time course, post-stimulus data for each cell were scaled to force the average of the last four measurements to 0.69. Continuous line, exponential giving best least-squares fit (time constant $6 \mathrm{~min})$. c
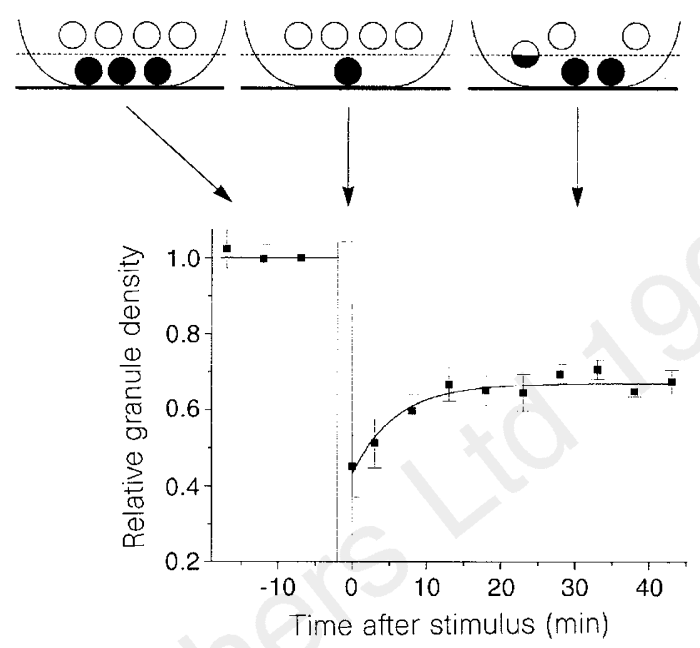

a

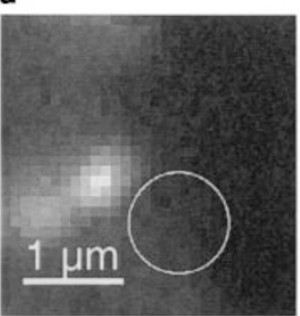

$t(\mathrm{~s})$

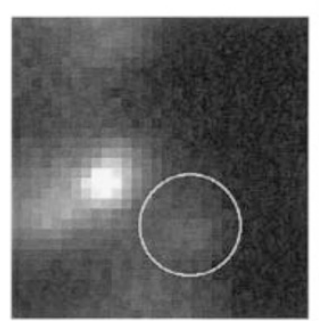

150

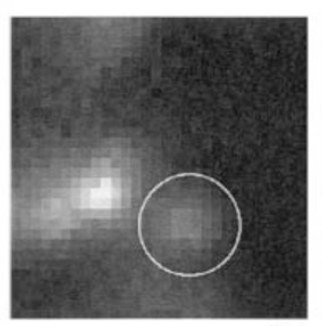

152

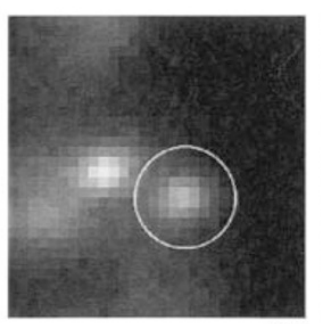

160

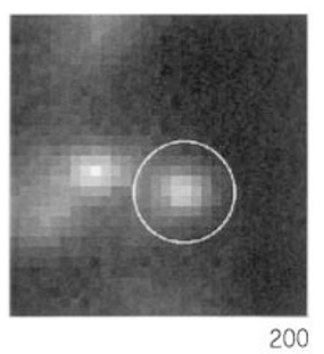

b

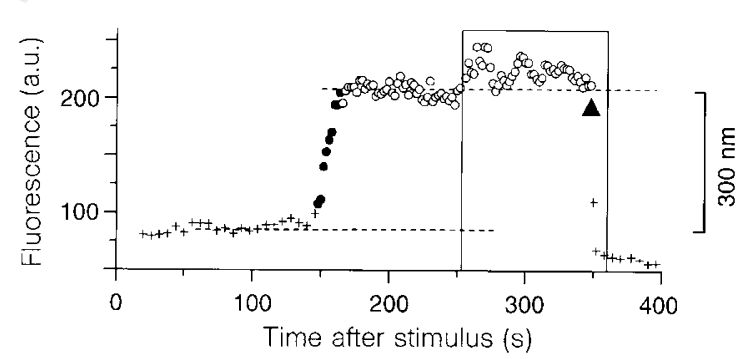

Figure $4 \mathbf{a}$, A granule appearing beneath the plasmalemma. Times shown are relative to the end of the first stimulus; circles outlining the granule are centred on its positions. b. Fluorescence intensity in the centre of the circles plotted against time. a.u., Arbitrary units. The initial fluorescence is background from neighbouring granules or from out-of-focus granules excited weakly by deeply penetrating photons of the evanescent wave. For the duration shown by the rectangle, $\left[\mathrm{K}^{+}\right]$ was elevated for a second time. Accompanying intensity fluctuations are due to a second granule approaching and retreating laterally (images not shown). Dashed lines: fluorescence before the granule appeared (lower) and immediately before it underwent exocytosis (arrowhead); they provide an approximate calibration for c
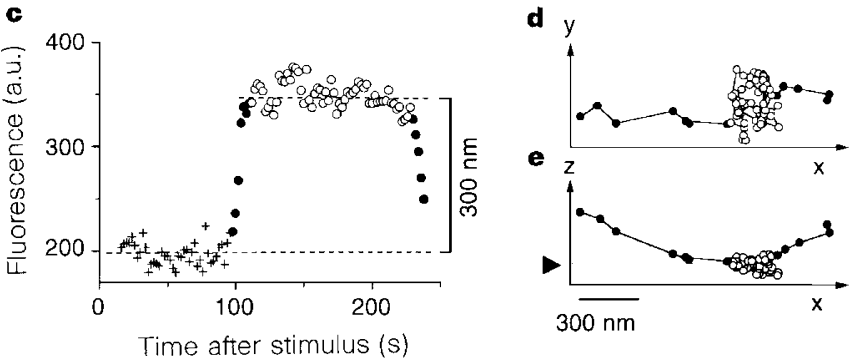
With plasmelemma and glass in contact (images as in Fig. 1g, h; results not shown), the approaching granule must have traversed the entire 300-nm layer where the evanescent wave makes it visible in our microscope (see Methods). With this distance calibration (dashed lines in Fig. 4b, c), the granule's vertical velocity (the component perpendicular to the plasmalemma) was $24 \mathrm{~nm} \mathrm{~s}^{-1}$, averaged from first appearance to $80 \%$ peak intensity ( $34 \pm 17 \mathrm{~nm} \mathrm{~s}^{-1} ; 8$ granules in 5 cells). In 14 approaching granules ( 6 cells), the median time to reach $80 \%$ of maximum brightness was $14 \mathrm{~s}$.

Figure $4 \mathrm{c}$ show the result of a similar analysis in the absence of a stimulus. This granule did not exocytose, but its fluorescence remained high and nearly constant as if it, too, had reached the plasmalemma. After $100 \mathrm{~s}$, the granule dimmed gradually as it moved back into the cytosol. Figure $4 \mathrm{~d}$ plots its lateral trajectory (that is, in the plane of the plasmalemma). The granule approached in a highly directed fashion over nearly $0.5 \mu \mathrm{m}$ (filled circles), apparently moving by means other than diffusion. If a diffusion coefficient is calculated from the average distance moved in the 2-s interval between frames in Fig. $4 \mathrm{~d}$, the chance of encountering the approach trajectory (filled circles) by diffusion is $4 \times 10^{-5}$. On arrival at the plasmalemma, directed motion stopped (open circles), suggesting that docking had occurred, and restarted when the granule detached again (filled circles). In all of 6 similar observations, including those shown in Fig. 4b, the lateral motility diminished on arrival at the plasmalemma and increased again if the granule detached, changing by $0.65 \pm 0.15 \log$ units or 4.5 -fold ( 5 cells). The loss of motility at the plasmalemma indicates hindrance by an added drag force, probably due to capture by a plasmalemmal docking site. The residual apparently random lateral motion (open circles in Fig. 4d) was analysed by measuring the mean distance moved in successive 8 -s intervals. It could be accounted for by a diffusion coefficient of $3.0 \pm 0.4 \times 10^{-4} \mu \mathrm{m}^{2} \mathrm{~s}^{-1}(n=6)$. The corresponding value for plasmalemma-resident granules in resting cells was similar $\left(3.0 \pm 0.8 \times 10^{-4} \mu \mathrm{m}^{2} \mathrm{~s}^{-1} ; 5\right.$ cells with $2-6$ granules each). Both values are 5,000 times smaller than they would be in water.

For a granule reaching the plasmalemma, its distance from the plasmalemma during approach or departure may be calculated from its intensity (Fig. 4c), and combined with the data in Fig. 4d to plot the trajectory in a vertical plane (Fig. 4e). The granule landed on the plasmalemma at an angle (Fig. 4e, filled circles), nearly stopped moving for $100 \mathrm{~s}$ after touch-down (open circles) and then detached and ascended back into the cytosol. Evidently, docking is reversible. Most approaching granules moved laterally as well as vertically, as in Fig. $4 \mathrm{c}-\mathrm{e}$. The average approach speed in all three dimensions was $40 \pm 16 \mathrm{~nm} \mathrm{~s}^{-1}$, with top speeds of $114 \pm 11 \mathrm{nms}^{-1}$ (9 granules in 6 cells; $\left.22-25^{\circ} \mathrm{C}\right)$. At higher temperatures, this top speed might approach that of actin-based organelle transport in neuronal growth cones $\left(480 \mathrm{~nm} \mathrm{~s}^{-1} ; 37^{\circ} \mathrm{C}\right.$; ref. 14). Like many other cells, chromaffin cells have a dense subplasmalemmal network of actin filaments ${ }^{15,16}$.

We have tracked single granules en route from the cytosol to their docking sites and hence to their exocytosis. In ultrastructural studies, a typical resting chromaffin cell had 1,000 secretory granules stuck to its plasmalemma. Most could exocytose during 2 min stimulation with elevated $\left[\mathrm{K}^{+}\right]$. Stimulated exocytosis was observed by evanescent-wave microscopy and seen to cause patches of empty plasmalemma. These were later repopulated with fresh granules, taking an average $6 \mathrm{~min}\left(22^{\circ} \mathrm{C}\right)$ to arrive. The pool of morphologically docked granules in chromaffin cells was much larger and was replenished far more slowly than the 'rapidly releasable' pool defined electrophysiologically ${ }^{8}$. Probably granules do not attain exocytic competence immediately after docking, and the docking complex must undergo subsequent maturation.

We conclude that granules reach the plasmalemma in a directed manner, moving actively. After arrival, granules lose lateral motility, consistent with the ultrastructural finding that they bind to the plasmalemma. Future experiments on permeabilized cells must identify the motors that carry granules to their docking sites, and address the molecular and functional mechanism of docking.

\section{Methods}

Bovine chromaffin cells ${ }^{17}$ were used 2 to 3 days after plating onto glass coverslips in buffer containing (in $\mathrm{mM}$ ) $135 \mathrm{NaCl}, 2 \mathrm{KCl}, 5 \mathrm{CaCl}_{2}, 2 \mathrm{MgCl}_{2}$, $10 \mathrm{Na}$-HEPES, pH 7.2, which kept the plasmalemma at a potential where calcium channels are shut and secretion does not occur. To stimulate secretion, $60 \mathrm{mM} \mathrm{KCl}$ replaced $\mathrm{NaCl}$ for $2 \mathrm{~min}$. All experiments were done at $22-25^{\circ} \mathrm{C}$; values are \pm s.e.

Electron microscopy. Cells were quickly frozen after $2 \mathrm{~min}$ in buffer with low or high $\left[\mathrm{K}^{+}\right]$, by plunging them into liquid ethane, and processed as described $^{17,18}$. Analysis was at 100,000 -fold magnification by counting peripheral granules per length of plasmalemma, as determined with a map measuring wheel. To convert the results into granules per $\mu \mathrm{m}^{2}$ of plasmalemma, they were divided by the sum of the section thickness $(100 \mathrm{~nm})$ plus the diameter of the structure being sampled ${ }^{19}$. This was $0.077 \mu \mathrm{m}$ for the contact zone between the membrane of a docked granule and the plasmalemma (defined as the distance over which both remained within $10 \mathrm{~nm}$; median, 194 granules), and $0.324 \mu \mathrm{m}$ for granules located more than one granule radius from the plasmalemma ${ }^{17}$. Light microscopy. Cells were stained for $15 \mathrm{~min}$ in buffer with $3 \mu \mathrm{M}$ acridine orange at $22^{\circ} \mathrm{C}$, then washed thoroughly in dye-free buffer and viewed 3090 min later (Zeiss 100×, 1.4 NA oil objective, 16-bit back-illuminated CCD camera; Princeton Instruments). Interference reflection images (IRM's) were taken with $600 \mathrm{~nm}$ light coupled into the epiillumination port with an appropriate short-pass dichroic mirror. To reduce stray reflection, we subtracted an image taken at the same focal plane but showing no cell. IRM images are darker the closer the plasmalemma approaches the coverslip; with our illumination aperture of about 1.2, all areas darker than background are expected to lie within $80 \mathrm{~nm}$ of the coverslip ${ }^{20}$.

Fluorescence images were taken with evanescent-wave excitation. We selected cells whose clear and dark 'footprint' in IRM indicated close adherence to the cover glass. We rejected about half of them because fluorescent spots were not clearly distinct and blended into each other. This selection probably excluded cells with high granule densities. 488-nm light from an argon laser was passed into the microscope's epifluorescence port. An annular mask, placed in a plane conjugate to the objective's back focal plane, blocked all but the most marginal rays ${ }^{7}$. These leave the objective at a supercritical angle and hence suffer total reflection at the coverslip/cell interface. Such epi-illumination arrangement allowed free access to the cell, so that secretion could be measured with a carbon fibre ( $8 \mu \mathrm{m}$ tip diameter at $0.7 \mathrm{~V}$; ref. 21 ) placed $1-2 \mu \mathrm{m}$ from the cell surface. To probe the evanescent wave, we held fluorescent $280 \mathrm{~nm}$ diameter beads (similar in size to chromaffin granules) at varying distances from the coverslip. This was done by placing a planoconvex lens curved-side down onto a coverslip, and letting beads adsorb to the surfaces of both. The vertical distance between beads at the two surfaces was measured with a calibrated piezoelectric focusing drive. When the objective was focused on beads at the coverslip, peak fluorescence from beads at the lens decreased roughly linearly with their distance from the coverslip. The regression line fell to zero at $311 \mathrm{~nm}$ (not shown). For counting granules and for tracking their paths in the plane of the membrane, images were processed as for Fig. 3b, and granule locations determined with an algorithm ${ }^{22}$ (Metamorph, Universal Imaging) that identified the centre of mass of a granule's fluorescence with a standard deviation of $<20 \mathrm{~nm}$ (tests with fluorescent beads). To compare lateral motilities in Fig. 4c, we divided the distance covered during the granule's approach (represented by the distance between the first and last filled circle) by that covered during an interval of the same duration but starting $20 \mathrm{~s}$ after the point represented by the first open circle (putative arrival at the plasmalemma). When granules approached slowly, we used only the last 10 locations during the approach. The fluorescence intensity of an individual granule was measured as the average over a $0.3 \times 0.3 \mu \mathrm{m}$ square placed in its centre. Granules were rejected if, during their stay near the plasmalemma (open circles), the root-mean square intensity fluctuation was $>10 \%$ of the fluorescence increase brought by the granule's arrival. 
Received 18 April; accepted 28 May 1997.

Broadie, K. et al. Syntaxin and synaptobrevin function downstream of vesicle docking in Drosophila. Neuron 15, 663-673 (1995).

2. Pieribone, V. A. et al. Distinct pools of synaptic vesicles in transmitter release. Nature 375, 493-497 (1995).

3. Ceccarelli, B. \& Hurlbut, W. P. Vesicle hypothesis of the release of quanta of acetylcholine. Physiol. Rev 60, 396-441 (1980).

4. Hunt, J. M. et al. A post-docking role for synaptobrevin in synaptic vesicle fusion. Neuron 12, 1269 1279 (1994).

Gillis, K. D. \& Chow, R. H. Kinetics of exocytosis in adrenal chromaffin cells. Sem. Cell Dev. Biol. (in the press).

6. Trifaro, J. M. \& Vitale, M. L. Cytoskeleton dynamics during neurotransmitter release. Trends Neurosci. 16, 466-472 (1993)

7. Stout, A. L. \& Axelrod, D. Evanescent field excitation of fluorescence by epillumination microscopy. Appl. Optics 28, 5237-5242 (1989).

8. Moser, T. \& Neher, E. Rapid exocytosis in single chromaffin cells recorded from mouse adrenal slices. $J$. Neurosci. 17, 2314-2323 (1997).

9. Stevens, C. F. \& Tsujimoto, T. Estimates for the pool size of releasable quanta at a single central synapse and for the time required to refill the pool. Proc. Natl Acad. Sci. USA 92, 846-849 (1995).

10. von Gersdorff, H. \& Matthews, G. Depletion and replenishment of vesicle pools at a ribbon-type synaptic terminal. J. Neurosci. 17, 1919-1927 (1997).

11. Schroeder, T. J., Jankowski, J. A., Senyshyn, J., Holz, R. W. \& Wightman, R. M. Zones of exocytotic release on bovine adrenal medullary cells in culture. J. Biol. Chem. 269, 17215-17220 (1994).

12. Ryan, T. A. \& Smith, S. J. Vesicle pool mobilization during action potential firing at hippocampa synapses. Neuron 14, 983-989 (1995).

13. Betz, W. J. \& Wu, L.-G. Kinetics of synaptic-vesicle recycling. Curr. Biol. 5, 1098-1101 (1995).

14. Evans, L. L. \& Bridgman, P. C. Particles move along actin filament bundles in nerve growth cones. Proc. Natl Acad. Sci. USA 92, 10954-10958 (1995).

15. Lee, R. W. \& Trifaro, J. M. Characterization of anti-actin antibodies and their use in immunocytochemical studies on the localization of actin in adrenal chromaffin cells in culture. Neurosci. 6, 20872108 (1981).

16. Cheek, T. R. \& Burgoyne, R. D. Nicotine-evoked disassembly of cortical actin filaments in adrenal chromaffin cells. FEBS Lett. 207, 110-114 (1986).

17. Parsons, T. D., Coorssen, J. R., Horstmann, H. \& Almers, W. Docked granules, the exocytic burst, and the need for ATP hydrolysis in endocrine cells. Neuron 15, 1085-1096 (1995).

18. Tse, F. W., Tse, A., Hille, B., Horstmann, H. \& Almers, W. Local $\mathrm{Ca}^{2+}$ release from internal stores controls exocytosis in pituitary gonadotrophs. Neuron 18, 121-132 (1997).

19. Weibel, E. R., Gonzague, S. K. \& Scherle, W. F. Practical stereological methods for morphometric cytology. J. Cell Biol. 30, 23-38 (1966)

20. Gingell, T. \& Todd, I. Interference reflection microscopy. A quantitative theory for image interpretation and its application to cell-substratum separation measurement. Biophys. J. 26, 507-526 (1979).

21. Chow, R. H. \& von Rüden, L. in Single-Channel Recording (eds Sakmann, B. \& Neher, E.) 245-275 (1995)

22. Ghosh, R. N. \& Webb, W. W. Automated detection and tracking of individual and clustered cell surface low density lipoprotein receptor molecules. Biophys. J. 66, 1301-1318 (1994).

23. Wightman, R. M. et al. Temporally resolved catecholamine spikes correspond to single vesicle release from individual chromaffin cells. Proc. Natl Acad. Sci. USA 88, 10754-10758 (1991).

24. Terakawa, S., Fan, J. H., Kumakura, K. \& Ohara-Imaizumi, M. Quantitative analysis of exocytosi directly visualized in living chromaffine cells. Neurosci. Lett. 123, 82-86 (1991)

Acknowledgements. We thank J. Howard, M. Lindau, W. M. Roberts and T. Soldati for their helpful suggestions on the manuscript.

Correspondence and requests for materials should be addressed to W.A. (e-mail: almers@mzf.mpimfheidelberg.mpg.de)

\section{Optical detection of a quantal presynaptic membrane turnover}

\section{Timothy A. Ryan $\star$, Harald Reuter $\dagger$ \& Stephen J Smith}

Department of Molecular and Cellular Physiology, Stanford University Medical School, Stanford, California 94305, USA

$\dagger$ Department of Pharmacology, University of Bern, 3010 Bern, Switzerland

Exploration of the mechanisms and plasticity of synaptic transmission has been hindered by the lack of a method to measure single vesicle turnover directly in individual presynaptic boutons at isolated nerve terminals. Although postsynaptic electrical recordings have provided a wealth of invaluable basic information about quantal presynaptic processes ${ }^{1}$, this approach has often proved difficult to apply at most central nervous system synapses $^{2-6}$. Here we describe the direct optical detection of single quantal events in individual presynaptic boutons of cultured hippocampal neurons. Using the fluorescent dye FM 1-43 as a tracer for presynaptic endocytosis ${ }^{7-10}$, we have characterized

Present address: Department of Biochemistry, Cornell University Medical College, 1300 York Avenue, New York, New York 10021, USA. both evoked and spontaneous components of presynaptic function at the level of individual quanta. Our results are consistent with quantal interpretations of previous electrophysiological analyses $^{1-6}$ and provide new information about the unitary membrane recycling event and its coupling to individual action potential stimuli, about spontaneous vesicle turnover at individual boutons, and about the numbers of vesicles recycling at individual boutons.

The strategy we used for optical detection of single presynaptic quanta builds upon earlier studies using the fluorescent dye FM $1-43$ as a marker for synaptic vesicle turnover ${ }^{7-10}$. So far there has been no direct evidence demonstrating that uptake and release of such a vesicle market can occur in quantized units corresponding to single vesicles. Our approach has been to include refinements designed for the measurement of very weak presynaptic fluorescence signals. Single-molecule fluorescence detection ${ }^{11-14}$ has shown that detection of weak signals is limited primarily by the presence of background fluorescence in instruments and specimens. We used confocal laser scanning microscopy to minimize instrumental autofluorescence background, and sparse monolayer cell cultures and the briefest possible staining exposures to FM 1-43 to minimize specimen fluorescence backgrounds.

A scheme of our experimental protocol is shown at the top of Fig. 1. Figure 1a is a Nomarski image illustrating the site of a putative axo-dendritic synaptic junction (arrow). Figure 1b, c shows fluorescence images of the same field, acquired sequentially after FM 1-43 loading with a single action potential (AP) stimulus (image A in the schematic): both panels show a small area of bright fluorescence localized to the junction site-measurement reproducibility is excellent and is generally sustained for hours at unstimulated boutons. Figure 1d, e shows reduction in fluorescence produced by a long unloading train of APs (image B). The fluorescence signal $\Delta F$ is defined as the difference in bouton fluorescence measurements before and after unloading (that is, images $\mathrm{A}-\mathrm{B}$ ). This $\Delta F$ signal presumably represents the amount of dye taken up into a releasable vesicular pool during the specified load. A second series (Fig. 1f-h) with more stimulation during loading (20 AP) verifies the presence of a viable presynaptic bouton at the site of interest. The 20 AP load resulted in 16-fold greater uptake of releasable dye (Fig. 1i).

To test for the postulated quantization of FM 1-43 signals, we measured fluorescence values across a large number of minimally stimulated individual boutons. Figure $2 \mathrm{a}$ represents individual measurements from a representative experiment in which just 1 AP was stimulated: each vertical set of points represents three separate measurements from the same bouton (fluorescence after loading but before unloading, black circles; the residual nonreleasable fluorescence measured after extensive unloading, white circles; and the intrinsic fluorescence of the synapse measured before loading $\left(F_{\mathrm{I}}\right)$, squares); solid, vertical lines represent $\Delta F$, and dotted lines represent amplitudes of the residual fluorescence signal $F_{\mathrm{R}}$. These data show that releasable fluorescence $(\Delta F)$ is $\sim 10$ times greater than $F_{\mathrm{I}}$, but that $F_{\mathrm{R}}$ magnitude is in the same range as the minimal $\Delta F$ signals. Because $F_{\mathrm{R}}$ became visible after even the briefest dye exposures, regardless of whether any APs are fired, this weak, non-releasable fluorescence probably reflects a residual membrane staining with no special relationship to synaptic vesicle recycling. This interpretation of $F_{\mathrm{R}}$ is further supported by the lack of any significant bouton-by-bouton correlation between $F_{\mathrm{R}}$ and $\Delta F$ values (data not shown).

Figure $2 \mathrm{~b}$ shows a histogram of the same $\Delta F$ values as in Fig. 2a. This histogram shows a distinct peak at a $\Delta F$ amplitude of 63 photoelectrons. There are also hints of secondary and tertiary peaks at double and triple that amplitude, but clearly the number of observations is inadequate for much certainty here. By pooling observations from several experiments, however, a multimodal response amplitude distribution becomes much more obvious. Figure 3a shows a frequency histogram of the different values of 